\title{
The time course of systems consolidation of spatial memory from recent to remote retention: A comparison of the Immediate Early Genes Zif268, c-Fos and Arc
}

\author{
Daniel N. Barry, Andrew N. Coogan, Sean Commins* \\ Department of Psychology, Maynooth University, Co. Kildare, Ireland
}

\section{A R T I C L E I N F O}

\section{Article history:}

Received 28 October 2015

Revised 21 December 2015

Accepted 24 December 2015

Available online 31 December 2015

\section{Keywords:}

Spatial navigation

Memory

Systems consolidation

Immediate Early Genes

\begin{abstract}
A B S T R A C T
Systems consolidation is a process involving the stabilisation of memory traces in the neocortex over time. The medial prefrontal cortex becomes increasingly important during the retrieval of older memories, however the timescale of its involvement is unclear, and the contribution of other neocortical brain regions to remote memory have received little attention. The Immediate Early Genes (IEGs) Zif268, c-Fos and Arc have been utilised as markers of neural activity during spatial memory retrieval, however the lack of a direct comparison between them hinders the interpretation of results. To address these questions, we examined the expression of Zif268, Arc and c-Fos protein in the medial prefrontal cortex, as well as the hippocampus, and the entorhinal, perirhinal, retrosplenial and parietal cortices of male Wistar rats following a probe trial of the Morris water maze either one day, seven days, 14 days or 30 days after acquisition. Activity of the medial prefrontal cortex during retrieval, as measured by all three IEGs, increased in correspondence with the age of the memory, reaching significance between 14 and 30 days. Similar increases in c-Fos and Arc were observed over the course of consolidation in other neocortical and parahippocampal areas, however this pattern was not observed with Zif268. Activity of the hippocampus remained largely unchanged across retention intervals. These findings suggest that systems consolidation of spatial memory takes at least two weeks, are consistent with an ongoing role for the hippocampus in the retrieval of spatial memory, and suggest that c-Fos and Arc may be a more sensitive measure of neural activity in response to behavioural tasks than Zif268.
\end{abstract}

(c) 2015 Elsevier Inc. All rights reserved.

\section{Introduction}

Newly acquired memories are thought to depend primarily on the hippocampus for their successful retrieval, whereas the neocortex assumes this responsibility over the course of time, in a process known as systems level consolidation (Frankland \& Bontempi, 2005). However, whether or not the role of the hippocampus in retrieving detailed episodic and spatial memory is time-limited (Squire, 1992) or permanent (Nadel \& Moscovitch, 1997) remains an open debate. There exists neuropsychological evidence to support both views (Squire \& Bayley, 2007; Winocur \& Moscovitch, 2011), and studies of spatial memory in animals have also proven inconclusive. Hippocampal lesions spare remote spatial discrimination memory (Maviel, Durkin, Menzaghi, \& Bontempi, 2004), but not allocentric spatial memory in the Morris water maze (Broadbent, Squire, \& Clark, 2006). Contextual fear memories can

\footnotetext{
* Corresponding author. Fax: +35317084767.

E-mail address: Sean.Commins@nuim.ie (S. Commins).
}

be abolished by pharmacological inactivation (Cullen, Gilman, Winiecki, Riccio, \& Jasnow, 2015) or optogenetic inhibition (Goshen et al., 2011) of area CA1 in the hippocampus for prolonged retention intervals of up to 12 weeks. Furthermore, the precision of remote spatial memory in the water maze, and contextual fear memory, is associated with the extent of long-term structural plasticity in area CA3 (Ruediger et al., 2011).

The neocortical structures supporting remote spatial memory have not been clearly defined, although inactivation of the anterior cingulate cortex disrupts remote memories while leaving recent memories intact (Teixeira, Pomedli, Maei, Kee, \& Frankland, 2006). However, this region may play a role in both recent and remote recall of spatial memory in the water maze (Leon, Bruno, Allard, Nader, \& Cuello, 2010). Therefore it is unclear how long it takes for newly acquired spatial memories to become dependent on extrahippocampal structures. Studies investigating systems consolidation in animals normally regard one and 30 days as recent and remote retention, respectively. However, attempts to disrupt spatial memory consolidation in first few weeks following 
learning suggest that this process may take place over a shorter timescale (Riedel et al., 1999; Shimizu, 2000; Frankland, O’Brien, Ohno, Kirkwood, \& Silva, 2001).

The expression of Immediate Early Genes (IEGs) such as Zif268, c-Fos and Arc is rapidly induced following neuronal activity, leading to structural and functional changes to the neuron which are essential for memory formation (Tischmeyer \& Grimm, 1999; Knapska \& Kaczmarek, 2004; Plath \& et al., 2006). IEG imaging allows for the visualisation of patterns of neural activity following learning, and has also been employed to investigate the activity of brain regions during memory retrieval (see Barry \& Commins, 2011). Hippocampal IEG expression decreases from recent to remote memory in a spatial discrimination task (Maviel et al., 2004), but remains elevated during allocentric remote spatial memory retrieval in the Morris water maze (Teixeira et al., 2006). Increases in IEG expression are observed from recent to remote retention in the anterior cingulate cortex for both tasks, however (Maviel et al., 2004; Teixeira et al., 2006). To date, one study has attempted to chart the increase in activity of cortical sites over the course of spatial memory consolidation using the IEG c-Fos as a marker of neural activity (Bonaccorsi et al., 2013), finding activity of the anterior cingulate cortex during a probe trial significantly increased as early as 10 days following learning.

IEG imaging studies often use just a single marker of neural activity, which can potentially hinder comparisons between findings and their interpretation. Here, we directly compared three IEGs, Zif268, c-Fos and Arc, to investigate the activity of hippocampal, parahippocampal and neocortical brain regions following a water maze probe trial at either one day, seven days, 14 days or 30 days following acquisition. By examining the extent of hippocampal and cortical engagement from recent to remote retention, we aimed to chart the time-course of systems consolidation of spatial memory, and investigate whether or not these three well-established markers of neural activity revealed similar patterns of expression.

\section{Materials and methods}

\subsection{Subjects}

Thirty-three male Wistar rats, obtained from Charles River Laboratories, UK, were used as subjects in this experiment. Subjects were approximately three months old and weighed 200-300 g at the beginning of experimentation. All animals were housed three per cage, in a temperature controlled environment $\left(21 \pm 1^{\circ} \mathrm{C}\right)$, which was maintained on a fixed $12: 12 \mathrm{~h}$ light-dark cycle (07:00-19:00). All rats were given ad libitum access to food and water. Experimentation took place during the light phase and all subjects were well handled before experimentation began. Guidelines for the maintenance and experimentation of animals conformed to the Department of Health and Children (Ireland) guidelines under statutory instrument (S.I.) No. 543 of 2012 and the European directive 2010/63/EU.

\subsection{Spatial task}

The Morris water maze (Morris, 1981), was chosen as the spatial task as it is an extensively studied and particularly demanding task of allocentric spatial memory. The water maze was made of black fibreglass $1.7 \mathrm{~m}$ in diameter and $36 \mathrm{~cm}$ in depth, mounted on a platform $70 \mathrm{~cm}$ above the floor. The maze was filled with water to a depth of $20 \mathrm{~cm}$ and maintained at a temperature of $20 \pm 1{ }^{\circ} \mathrm{C}$. The escape platform was $13.5 \mathrm{~cm}$ in width and $18 \mathrm{~cm}$ in height, and was placed in the centre of the northeast quadrant of the maze, submerged $2 \mathrm{~cm}$ below the surface of the water. The water maze was surrounded by a black curtain which obscured the rest of the room from view. Three distal cues were available, two $25 \mathrm{~W}$ bulbs which were suspended from the ceiling at a distance of $75 \mathrm{~cm}$ from the edge of the pool and at an angle of $60^{\circ}$, one in the north-east and the other in the south-east, and a rectangular piece of white card $(55 \mathrm{~cm} \times 81 \mathrm{~cm})$ which was also suspended from the ceiling on the west side of the maze. The animal's movements for each trial were recorded by a camera positioned directly above the centre of the maze. This information was collected by the digital tracking software EthoVision (Noldus Information Technologies, Wageningen, Netherlands).

Rats were randomly allocated to one of four experimental groups ( $n=7$ per group). Animals were trained for five consecutive days in the water maze, with four trials per day (see Harvey et al., 2008). Animals were placed into the water maze from one of four starting positions, either north, south, east or west, with each starting position used just once per day. Animals were allowed $60 \mathrm{~s}$ to locate the escape platform, after which they would be guided to the platform by the experimenter, and allowed to remain there for $15 \mathrm{~s}$. Following an inter-trial interval of $10 \mathrm{~s}$, the animal was placed back into the maze to begin the next trial. The limitations of using "free-swimming", as well as task-based controls as a comparison group in the water maze have been previously reported, with these control groups often displaying higher IEG expression than experimental groups in widespread brain regions (Shires \& Aggleton, 2008). This neural activity is likely due to factors such as increased stress (Duncan, Johnson, \& Breese, 1993; Cullinan, Herman, Battaglia, Akil, \& Watson, 1995; Ons, Marti, \& Armario, 2004) and incidental learning about the environment through exploration (Guzowski, McNaughton, Barnes, \& Worley, 1999), which can hinder interpretation of results. The present experiment circumvented these challenges by comparing relative levels of IEG expression from one time-point to another, as all experimental groups would be affected equally by any factor extraneous to the task which may influence expression. A naïve control group $(n=5)$ which was not exposed to the water maze apparatus was included to provide a baseline measure of IEG expression. The four spatially-trained groups were given a single retention probe trial, however the length of time between the final acquisition day and retention differed for each group. Probe trials took place either one day, seven days, 14 days or 30 days post-acquisition. The animals were placed back into the water maze from a south-west starting position, with the escape platform removed from the maze. Animals were allowed 60 s to search the maze for an escape. Successful retention of the maze was assessed by analysis of time spent searching in a circular area around where the platform was previously located, comprising $7 \%$ of the total searchable area of the maze, compared to equivalent areas of the maze. This target area was chosen for analysis as it was deemed a more accurate measure of successful retention than quadrant analysis (Moser, Moser, \& Andersen, 1993).

\subsection{Immunohistochemistry}

Immunohistochemical protocol for the detection of Zif268, cFos and Arc protein was carried out on all groups of animals. For the experimental groups, $90 \mathrm{~min}$ after the retention probe trial, rats were deeply anaesthetised with an intraperitoneal injection of sodium pentobarbital ( $100 \mathrm{mg} / \mathrm{kg}$, Euthatal), and subsequently perfused transcardially with ice cold $0.9 \%$ phosphate buffered saline (PBS, Ph7.4), followed by $4 \%$ paraformaldehyde in $0.1 \mathrm{M}$ phosphate buffer (PB, Ph7.4). Brains were then rapidly removed and post-fixed in $4 \%$ paraformaldehyde overnight, and then transferred to a $30 \%$ sucrose solution and stored at $4{ }^{\circ} \mathrm{C}$. Coronal sections were cut at $40 \mu \mathrm{m}$ using a freezing microtome, with every fourth section taken for subsequent immunohistochemical analysis. Rats in the 
caged control group were taken directly from their home cages and sacrificed.

Prior to the immunohistochemical procedure, free floating sections were stored in $0.1 \mathrm{M} \mathrm{PB}$ containing $0.01 \%$ sodium azide at $4{ }^{\circ} \mathrm{C}$. In order to minimise variation due to the immunohistochemical procedure, brain sections representing a particular region from all subjects were processed in a single batch. Sections were given two $10 \mathrm{~min}$ washes in $0.1 \mathrm{MPB}$, followed by a $10 \mathrm{~min}$ wash in $0.1 \mathrm{M}$ PB containing $0.2 \%$ Triton-X-100 (PBX). A 20 min wash in $0.1 \mathrm{M}$ PB containing $1.5 \%$ hydrogen peroxide was then carried out. This was followed by another two washes in $0.1 \mathrm{M} \mathrm{PB}$ and one in PBX. Sections were then blocked for $60 \mathrm{~min}$ at room temperature in 5\% normal goat serum (NGS) in $0.1 \mathrm{M} \mathrm{PBX}$. Sections were then incubated overnight in a primary antibody solution (2\% NGS in 0.1 M PBX). Labelling of Zif268, c-Fos and Arc were performed using the following primary antibodies: Zif268/Egr-1, rabbit polyclonal antibody raised against the $C$ terminus of human Egr-1 (dilution 1:3000; Santa Cruz Biotechnology); c-Fos, rabbit polyclonal antibody raised against the amino terminus of human cFos (dilution 1:2000; Santa Cruz Biotechnology); Arc, rabbit polyclonal antibody corresponding to amino acids 1-300 of Arc of human origin (dilution 1:800; Santa Cruz Biotechnology). After incubation with the primary antibody, sections were washed twice in $0.1 \mathrm{M}$ PB and once in PBX and then incubated with biotinylated secondary antibody (goat anti-rabbit, Jackson Laboratories, dilution 1:400) for $70 \mathrm{~min}$. Sections were again washed twice in $0.1 \mathrm{M}$ PB and once in $0.1 \mathrm{M}$ PBX before incubation with avidinbiotin-peroxidase complex (0.4\%; Vector Laboratories) for $90 \mathrm{~min}$ in lightproof conditions at room temperature. Sections were then given two 10 min washes in PB followed by one 10 min wash in $0.1 \mathrm{M}$ sodium acetate, $\mathrm{pH}$. This was followed by visualisation of the antigen using the nickel DAB method with glucose oxidase (Sigma, Poole, UK) as the catalyst. Sections were reacted for standardised lengths of time to ensure similar staining intensity across experimental groups. Sections were then mounted onto gelatinecoated slides, dried, dehydrated, cleared in Histoclear (National Diagnostics, Hull, UK), and coverslipped using Eukitt (Sigma, Poole, UK).

\subsection{Regions of interest}

Based on the findings of lesion and imaging studies, a number of regions were chosen for analysis due to their likely involvement in spatial memory processes. These included the dorsal hippocampus (CA1, CA3, dentate gyrus), parahippocampal areas (lateral and medial entorhinal, and perirhinal cortices), the medial prefrontal cortex (anterior cingulate, prelimbic and infralimbic cortices), and the retrosplenial and parietal cortices.

\subsection{Image analysis}

Counts of IEG expressing cells were calculated using an automated computerised cell counting procedure, which eliminated bias which could arise from manual counting procedures. Images were taken of the 11 regions sampled using an Olympus DP12 digital camera, mounted on an Olympus BX51 microscope and captured using a $4 \times$ magnification. As this level of magnification usually sampled a larger area than that under investigation, in order to obscure adjacent brain regions during image acquisition, appropriately scaled images of the coronal sections in question, adapted from Paxinos and Watson (2007), were printed onto clear acetate, with all regions except those of interest blacked out, and these were positioned over the brain section during image acquisition. This approach allowed for maximal coverage of the area of interest. This procedure was carried out for all regions sampled except the hippocampus, where images were manually cropped following image acquisition. Numbers of immunopositive cells were analysed using the public domain program Image (National Institute of Health, USA). Cell counts above a pre-defined brightness intensity threshold, and within a pre-defined particle area size were calculated. Counts from consecutive sections were averaged to produce a mean for each animal. As all animals were processed as a single batch during the immunohistochemical procedure, a normalisation procedure was not necessary and raw values were used for statistical analyses (Albasser, Poirier, \& Aggleton, 2010).

\subsection{Statistical analysis}

All statistical analyses were carried out using SPSS (Version 20). Acquisition of the water maze was evaluated using a mixed-design ANOVA with day as the repeated-measures factor and retention group as the within-group measure. Subsequent analyses of group escape latencies were assessed using a one-way repeatedmeasures ANOVA with Bonferroni correction. Time spent searching in the target area of the maze during probe trial was evaluated using a one-way repeated-measures ANOVA with Bonferroni correction. The significance of differences in IEG expression between groups was assessed using one-way ANOVA with Tukey post-hoc tests.

\section{Results}

\subsection{Acquisition}

The escape latencies of all four experimental groups were analysed to ensure comparable performance during acquisition. An overall significant effect for acquisition day was found (mixed ANOVA, $\left.F_{(4,96)}=36.22, p<0.001\right)$. Escape latencies for all four groups were significantly faster on day five than on day one (one-way ANOVAs, all $p<0.05$ ). There was no overall difference found in escape latencies between the groups, (mixed ANOVA, $F_{(3,24)}=1.33, p=0.288$ ), nor was an interaction effect between day and group observed, $\left.F_{(12,96)}=0.56, p=0.873\right)$. In summary, all four groups acquired the water maze task, demonstrating comparable performance (see Fig. 1A).

\subsection{Retention}

To assess successful retention of the water maze during the probe trial, time spent searching in a circular area around the target platform location, comprising $7 \%$ of the total maze area, was compared with equivalent areas in the remaining three maze quadrants. In the one day retention group, a main effect for platform area was found, (one-way repeated-measures ANOVA, $\left.F_{(1.42,8.49)}=13.38, p=0.004\right)$, with Bonferroni post-hoc tests confirming this group spent more time in the target platform area than the adjacent right ( $p=0.022$ ) or opposite $(p=0.001)$ area. An effect for platform area was also found in the seven day retention group, (one-way repeated-measures ANOVA, $F_{(3,18)}=13.49, p<0.001$ ), with rats in this group spending more time searching in the target area than the adjacent right $(p=0.024)$ or opposite $(p<0.001)$ area. In the 14 day retention group a main effect for platform area was observed, (one-way repeated-measures ANOVA, $F_{(3,18)}=9.31$, $p<0.001$ ), with Bonferroni post-hoc tests confirming this group spent more time searching in the target platform area than in the equivalent adjacent right $(p=0.024)$ or opposite $(p=0.029)$ area. A significant effect for platform area was also found in the 30 day retention group, (one-way repeated-measures ANOVA, $F_{(3,18)}=6.05, p=0.005$ ), with Bonferroni post-hoc tests revealing this group spent more time in the target platform area than the 

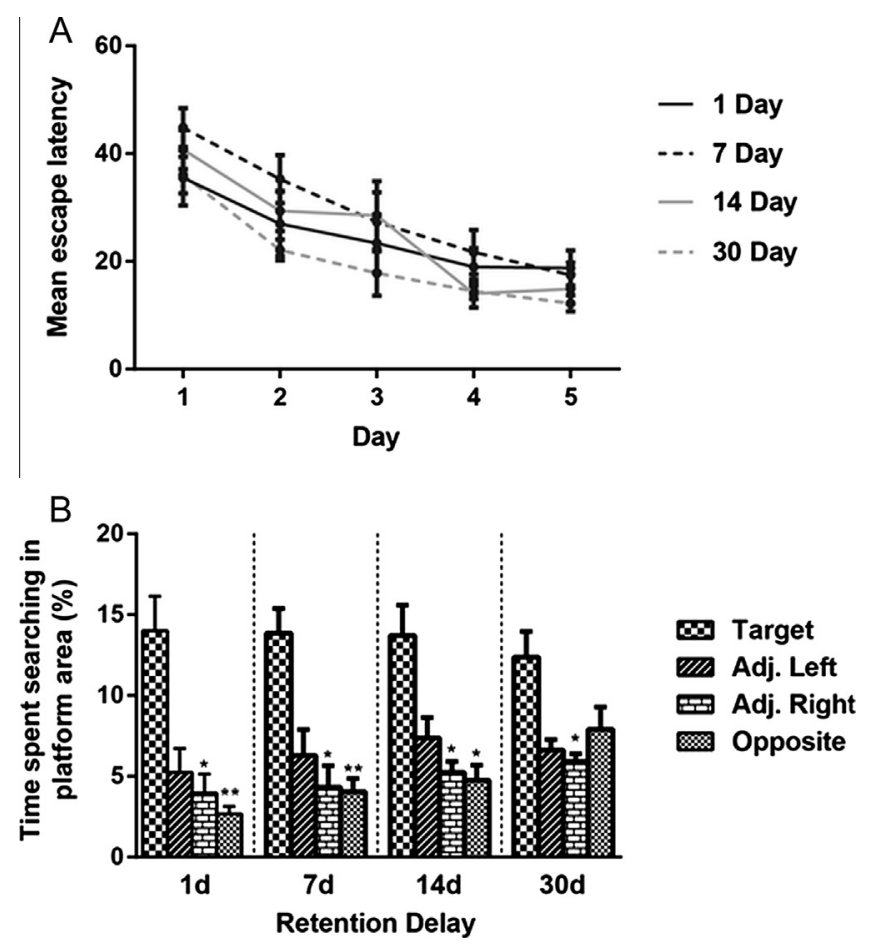

Fig. 1. Average daily escape latency in seconds $( \pm S E M)$ for all four water maze trained groups over five days of training $(a)$, and percentage time $( \pm$ SEM) searching in the target platform area versus equivalent areas in other quadrants in the water maze during a probe trial for the four retention groups (b). Asterisks indicate a significant difference between time spent in the target quadrant versus equivalent quadrants $\left({ }^{*} p<0.05 ;{ }^{* *} p<0.01\right)$.

adjacent right area $(p=0.029)$. All four groups therefore demonstrated successful retrieval of the task (see Fig. 1B).

\subsection{IEG analysis}

\subsubsection{Zif 268}

Zif268 counts were compared across the groups in all brain regions studied, however significant differences between experimental groups were limited to the medial prefrontal cortex. In the medial prefrontal cortex, a main effect for group was found in the anterior cingulate cortex, (one-way ANOVA, $F_{(4,26)}=2.97$, $p=0.038$ ), with Tukey post-hoc tests revealing that levels of Zif268 were significantly higher in the 30 day retention group compared to the one day group ( $p=0.037$ ). Zif268 expression also differed across groups in the prelimbic cortex (one-way ANOVA, $F_{(4,27)}=3.11, \quad p=0.032$ ), with Tukey post-hoc tests showing increased expression in the 30 day group compared to the one day group $(p=0.021)$. A difference across groups was also found in the infralimbic cortex, (one-way ANOVA, $F_{(4,25)}=8.31$, $p<0.001)$, with Tukey post-hoc tests revealing Zif268 counts were higher at 14 day $(p=0.003)$ and 30 day $(p<0.001)$ than one day retention. Zif268 counts were also significantly higher in the 30 day group compared to the seven day group $(p=0.008$, see Fig. 2A).

Limited differences were found in the hippocampus, where a main effect for group was not found in CA1 (one-way ANOVA, $F_{(4,26)}=2.65, p=0.056$ ), or CA3, (one-way ANOVA, $F_{(4,27)}=1.85$, $p=0.149$ ). A difference was found across groups in the dentate gyrus, (one-way ANOVA, $F_{(4,28)}=3.18, p=0.028$ ), with Tukey post-hoc tests revealing levels of Zif268 expression were lower in the one day retention group than caged controls $(p=0.012$, see Fig. 2B).
An overall effect for group was not observed in the lateral entorhinal (one-way ANOVA, $F_{(4,28)}=1.74, p=0.170$ ), or medial entorhinal (one-way ANOVA, $F_{(4,26)}=0.89, p=0.484$ ) cortices. While a main effect for group was found in the perirhinal cortex, (one-way ANOVA, $F_{(4,27)}=2.73, p=0.049$ ), Tukey post-hoc tests did not reveal any significant differences between the groups (see Fig. 2C). An overall effect for group was not observed in either the retrosplenial (one-way ANOVA, $F_{(4,28)}=0.47, p=0.757$ ) or parietal (one-way ANOVA, $F_{(4,27)}=1.69, p=0.182$ ) cortices (see Fig. 2D).

\subsection{2. c-Fos}

Levels of c-Fos for all groups were also analysed, revealing a more extensive pattern of overall differences between groups than the Zif268 analyses. A main effect for group was found in the anterior cingulate cortex (one-way ANOVA, $F_{(4,24)}=3.72, p=0.017$ ), but while Tukey post-hoc tests did not reveal any differences between the groups, the increase in c-Fos expression observed in this region between one day and 30 days approached statistical significance $(p=0.055)$. An overall effect for group was also found in the prelimbic cortex, (one-way ANOVA, $F_{(4,25)}=8.49, p<0.001$, with Tukey post-hoc tests confirming levels of c-Fos were significantly higher in the 14 day group than one day retention $(p=0.026)$ and caged controls $(p=0.041)$. The 30 day retention group had higher $c-F o s$ counts than one day retention $(p<0.001)$, seven day retention $(p=0.049)$, and caged controls $(p=0.001)$. A main effect for group was also found in the infralimbic cortex, (one-way ANOVA, $\left.F_{(4,26)}=18.06, p<0.001\right)$, with Tukey post-hoc tests revealing levels of c-Fos were higher in the 14 day $(p<0.001)$ and 30 day $(p<0.001)$ groups than the one day retention group. Counts were also higher in the 14 day $(p=0.042)$ and 30 day $(p=0.001)$ groups than the seven day retention groups. The 14 day $(p<0.001)$ and 30 day $(p<0.001)$ retention groups also had significantly higher counts than caged controls (see Fig. 3A).

A significant difference in c-Fos expression across groups was found in CA1, (one-way ANOVA, $F_{(4,26)}=4.02, p=0.011$ ), with Tukey post-hoc tests revealing counts were higher in the seven day $(p=0.049)$ than the one day retention group. A main effect for group was found in CA3, (one-way ANOVA, $F_{(4,26)}=5.03$, $p=0.004)$, with Tukey post-hoc tests showing c-Fos counts were significantly higher in the seven day $(p=0.041)$ and 14 day $(p=0.020)$ than one day retention groups. c-Fos counts in the 14 day retention group were also higher than caged control levels $(p=0.027)$. A significant difference across groups was also found in the dentate gyrus, (one-way ANOVA, $F_{(4,27)}=3.34, p=0.024$ ), with Tukey post-hoc tests revealing c-Fos counts were higher in the seven day than the one day retention group ( $p=0.041$, see Fig. $3 \mathrm{~B}$ ).

A main effect for group was found in the lateral entorhinal cortex (one-way ANOVA, $F_{(4,25)}=4.38, p=0.008$ ), with Tukey post-hoc tests revealing counts of $c$-Fos were significantly higher in the seven day than the one day retention group $(p=0.040)$ and caged controls $(p=0.027)$. A significant difference across groups was also found in the medial entorhinal cortex, (one-way ANOVA, $\left.F_{(4,25)}=3.90, p=0.014\right)$, with Tukey post-hoc tests revealing levels of c-Fos were higher in the 14 day retention group than caged controls $(p=0.047)$. A main effect for group was also found in the perirhinal cortex, (one-way ANOVA, $F_{(4,27)}=8.47, p<0.001$, with Tukey post-hoc tests showing c-Fos counts were higher in the 14 day than the one day retention group $(p=0.005)$ and caged controls $(p<0.001)$. $c$-Fos counts were also higher in this region in the 30 day than one day retention group $(p=0.016)$ and caged controls $(p=0.002)$. The seven day retention group also had higher $c-F o s$ counts than caged controls ( $p=0.046$, See Fig. $3 C$ ).

A significant difference was found across groups in the retrosplenial cortex, (one-way ANOVA, $F_{(4,24)}=4.14, p=0.011$ ), with Tukey post-hoc tests revealing c-Fos counts were higher in the 

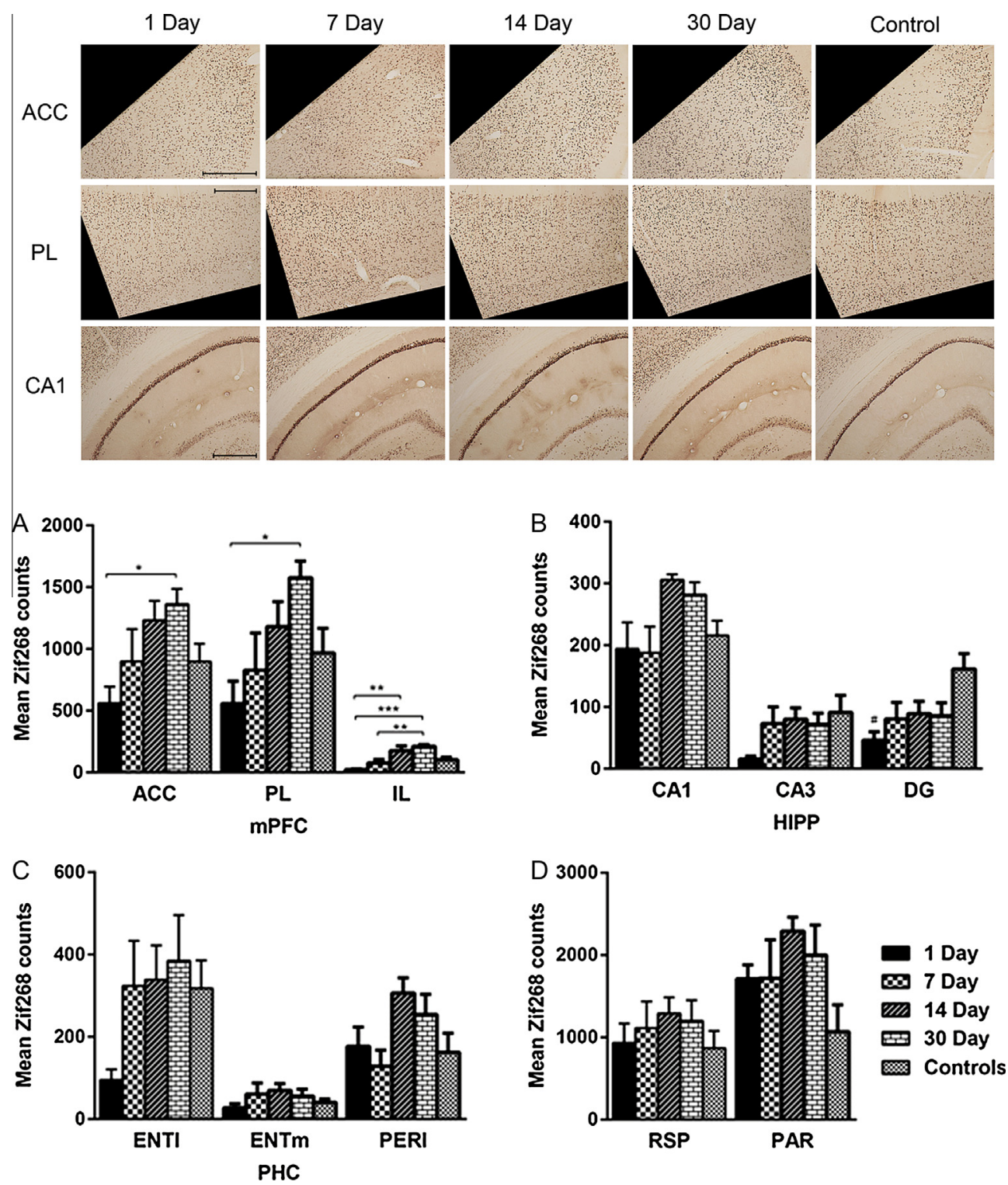

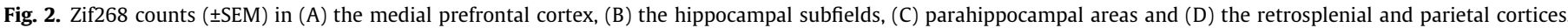

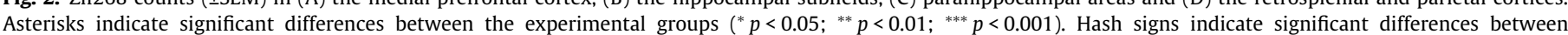

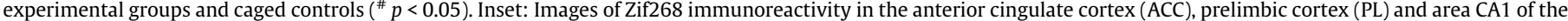
hippocampus in the four retention groups and caged controls. Scale bar $=500 \mu \mathrm{m}$.

30 day retention group than the caged control group $(p=0.039)$. A main effect for group was also found in the parietal cortex, (oneway ANOVA, $\left.F_{(4,27)}=4.07, p=0.010\right)$, with Tukey post-hoc tests confirming c-Fos counts were higher in the 14 day than the one day retention retention group $(p=0.029)$ and caged controls $(p=0.016$, see Fig. 3D).

\subsection{3. $\operatorname{Arc}$}

Levels of Arc expression during retention differed dynamically as a function of time since acquisition, demonstrating a similar pattern to the c-Fos analyses. A main effect for group was found in the anterior cingulate cortex (one-way ANOVA, $F_{(4,25)}=5.37, p=0.003$ ), with Tukey post-hoc tests revealing levels of Arc were significantly higher in the 30 day than the one day retention group $(p=0.012)$, and caged controls $(p=0.018)$. A significant difference across groups was found in the prelimbic cortex, (one-way ANOVA, $\left.F_{(4,25)}=5.95, p=0.002\right)$, with Tukey post-hoc tests confirming levels of Arc were significantly higher in the 14 day $(p=0.017)$ and 30 day retention groups $(p=0.013)$ than the one day retention group. Arc counts were also significantly higher in this region in the 14 day $(p=0.036)$ and 30 day $(p=0.029)$ retention groups than caged controls. A significant difference across groups was also observed in the infralimbic cortex, (one-way ANOVA, $F_{(4,26)}=4.30, p=0.008$ ), with Tukey post-hoc tests revealing levels of Arc were significantly higher in the 30 day than the one day retention group $(p=0.021)$ and caged controls $(p=0.039$, see Fig. 4A).

A significant difference across groups was found in CA3, (oneway ANOVA, $F_{(4,28)}=4.19, p=0.009$ ), with Tukey post-hoc analyses showing Arc counts were significantly higher at in the 14 day than the one day retention group $(p=0.013)$, and caged controls $(p=0.034)$. A main effect for group was not observed in the dentate gyrus, (one-way ANOVA, $F_{(4,27)}=1.46, p=0.241$, see Fig. $4 \mathrm{~B}$ ). Arc staining was weak or absent in CA1 therefore this region is not included in the Arc analyses.

There was a main effect for group found in the lateral entorhinal cortex, (one-way ANOVA, $F_{(4,24)}=5.26, p=0.003$ ), with Tukey posthoc tests revealing counts of Arc were significantly higher in the 

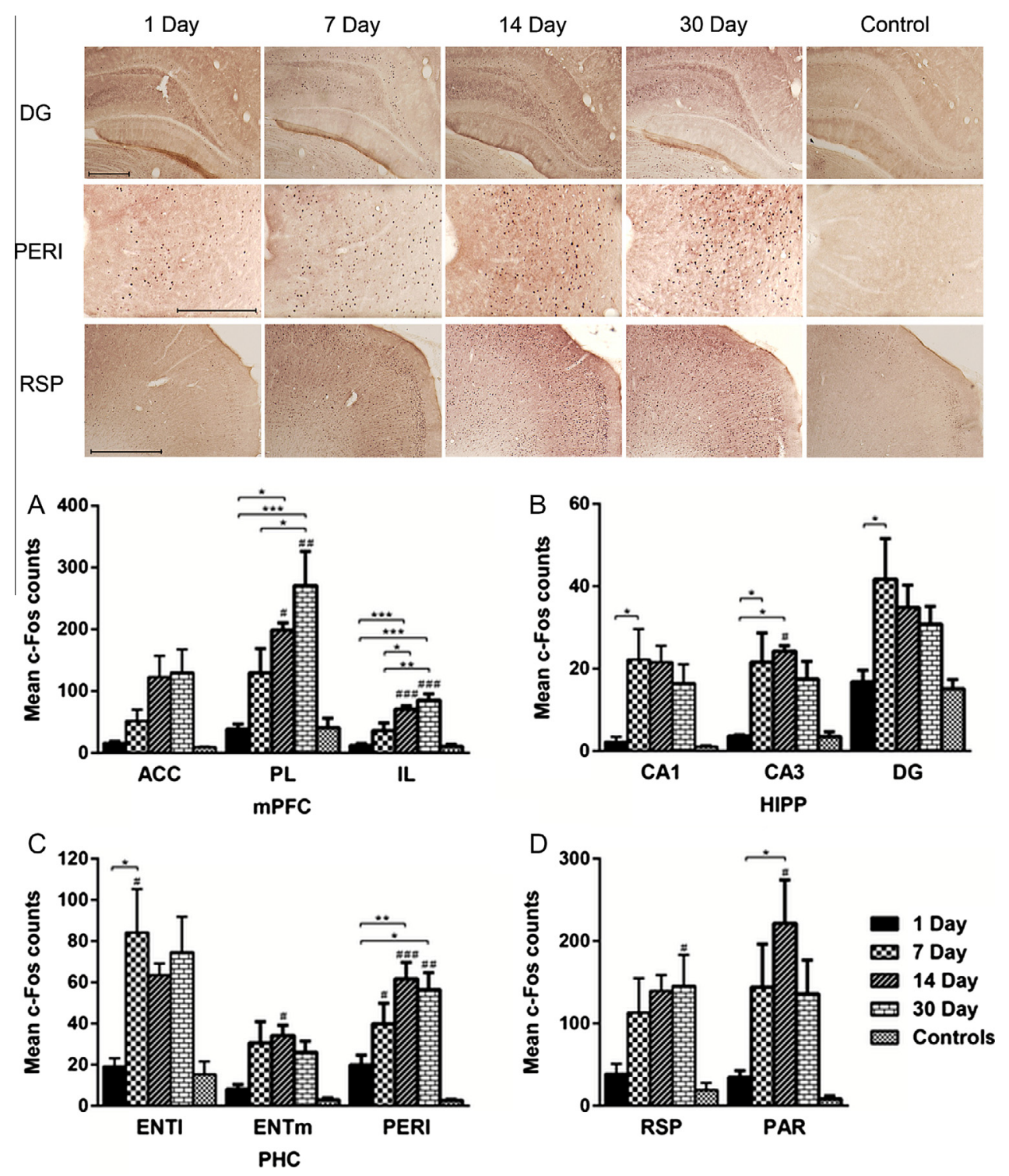

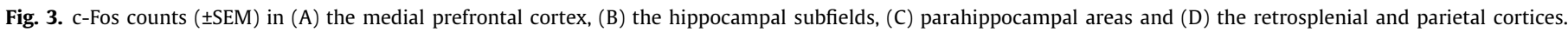

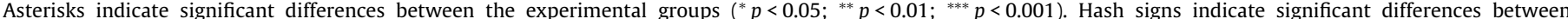

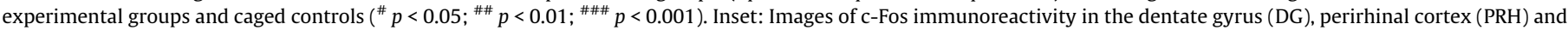
the retrosplenial cortex (RSP) in the four retention groups and caged controls. Scale bar $=500 \mu \mathrm{m}$.

30 day than the one day retention group ( $p=0.015)$ and caged controls $(p=0.016)$. A significant difference was not found across groups in the medial entorhinal cortex, (one-way ANOVA, $\left.F_{(4,26)}=2.31, p=0.85\right)$. A main effect for group was observed in the perirhinal cortex, (one-way ANOVA, $F_{(4,26)}=13.71, p<0.001$ ), with Tukey post-hoc tests revealing Arc counts were significantly higher in the 14 day $(p<0.001)$ and 30 day $(p=0.011)$ retention groups than the one day retention group. Arc counts were also significantly higher in the 14 day $(p<0.001)$ and 30 day $(p=0.043)$ retention groups than in the seven day retention group (see Fig. 4C).

A main effect for group was observed in the retrosplenial cortex, (one-way ANOVA, $F_{(4,26)}=3.63, p=0.018$ ), however Tukey post-hoc tests did not detect any significant differences between the groups. A significant difference across groups was found in the parietal cortex, (one-way ANOVA, $F_{(4,24)}=8.17, p<0.001$ ), with Tukey post-hoc tests revealing Arc counts were higher in the 14 day retention group than the one day $(p<0.001)$, and seven day retention groups $(p=0.002)$ and caged controls $(p=0.002)$. Arc counts in this region were also higher in the 30 day than the 14 day retention group $(p=0.040$, see Fig. 4D).

\section{Discussion}

The present study demonstrates, through multiple markers of neural activity, that the expression of newly acquired spatial memory results in increased activity of extrahippocampal structures between 14 and 30 days following learning. A wide range of neocortical and parahippocampal brain regions appeared to become increasingly recruited in parallel as the delay between acquisition and retention increases. These findings also emphasise an ongoing role for the hippocampus in the retrieval of spatial memory. Taking into consideration the consistent patterns which emerged from the analysis of c-Fos and Arc expression, the results suggest they may be a more sensitive marker of increased neural activity during spatial memory retention than Zif268. 

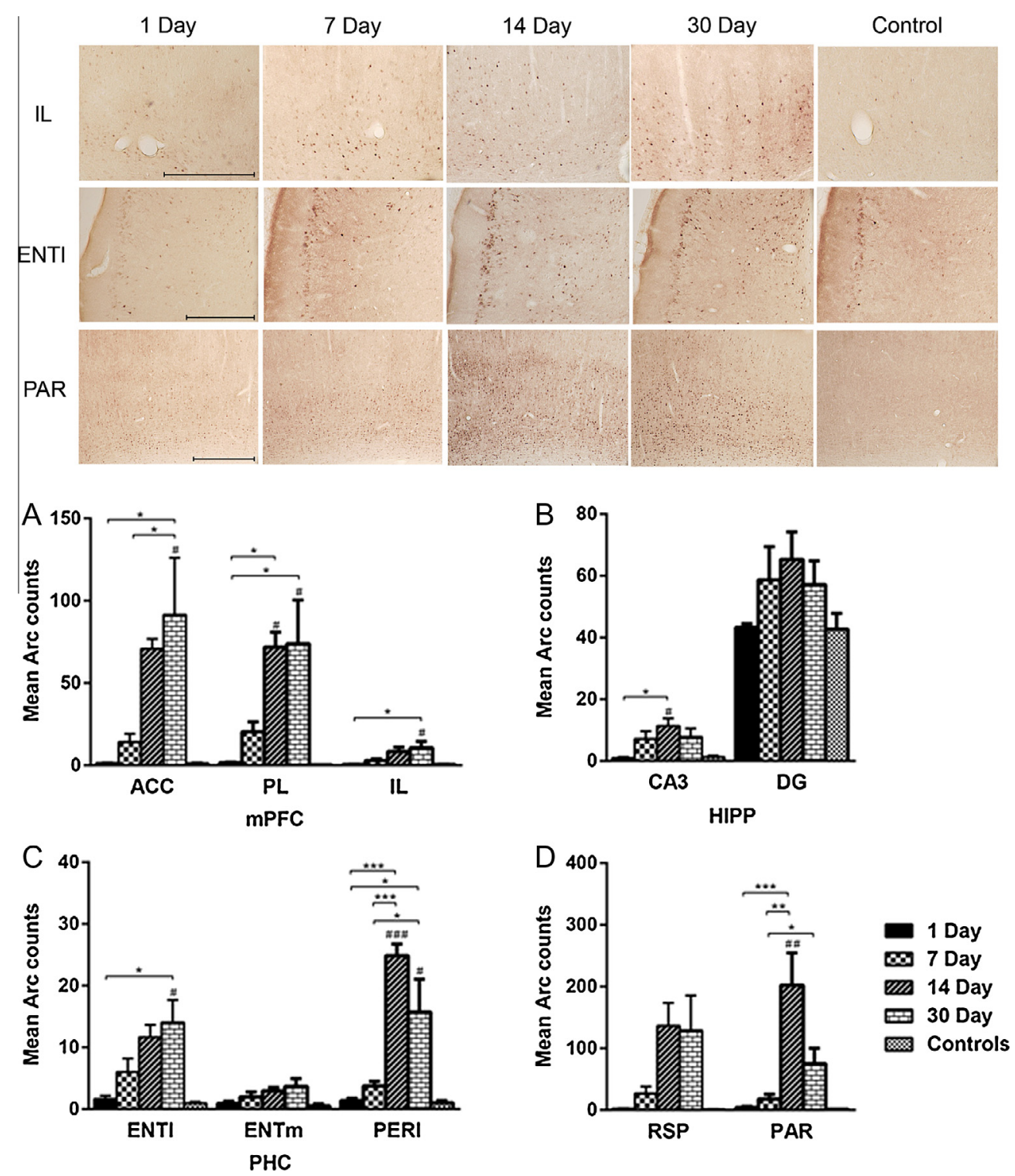

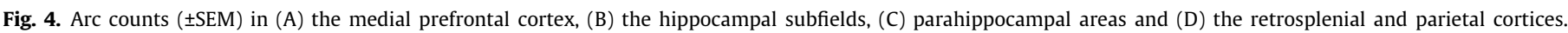

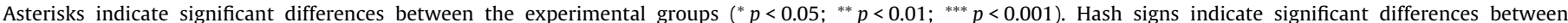

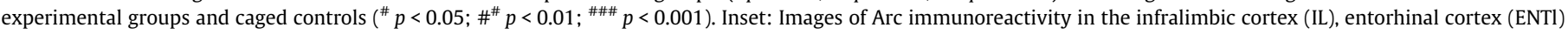
and the parietal cortex (PAR) in the four retention groups and caged controls. Scale bar $=500 \mu \mathrm{m}$.

Numerous studies have implicated the medial prefrontal cortex in remote spatial memory (Maviel et al., 2004; Teixeira et al., 2006; Lopez et al., 2012), and the present results have yielded a remarkable consistency across three different markers of neural activity in this region from recent to remote retention. The pattern which emerged was a gradual and linear increase in IEG activity which reached statistical significance between 14 and 30 days following learning, suggesting spatial memory takes at least two weeks to become dependent on extrahippocampal structures. The results are consistent with both standard consolidation theory which proposes a time-dependent reorganisation of memory within the brain (Squire, 1992), and also multiple trace theory/memory transformation theory which proposes that a schematic version of the memory is formed in the neocortex over time (Winocur \& Moscovitch, 2011). There is preliminary evidence that a schematic spatial memory is formed at some stage between one and 30 days following learning in the water maze (Richards et al., 2014), and the present results suggest that this may take the entire 30 day period for this schematic memory to be formed. Evidence for this region supporting a "gist-like" representation during remote memory has been observed in contextual fear memory, where pharmacological inactivation of the anterior cingulate cortex prevents the generalisation of a fear response from one environment to another during remote memory (Cullen et al., 2015). A competing theory of medial prefrontal cortex functioning proposes that it does not store information relevant to the task, rather it is involved in the retrieval of information from other areas of the brain when the memory trace becomes weaker over time (Rudy, Biedenkapp, \& O'Reilly, 2005), however there is evidence that this region undergoes structural changes over the course of consolidation, thereby displaying the characteristics of a long-term storage site (Maviel et al., 2004; Restivo, Vetere, Bontempi, \& Ammassari-Teule, 2009; Vetere et al., 2011). The medial prefrontal cortex has also been proposed to function as an integrator of incoming information, and the selection of an appropriate response based on previous experience (Euston, Gruber, \& McNaughton, 2012; Weible, 2013), and the 
present results suggest that this function may become increasingly important over time. Of note, the pattern of increased activity over time observed in the anterior cingulate cortex is reflected by a similar increase in the prelimbic and infralimbic cortex between recent and remote retention, suggesting these subregions make an equivalently important contribution to spatial memory retention.

Theories of systems consolidation differ with regards to the role of the hippocampus in long-term memory retention. Standard consolidation theory maintains that the involvement of the hippocampus in the retrieval of declarative memory is time-limited (Squire, 1992), whereas multiple trace theory argues that the retrieval of detailed contextual memory such as spatial memory will always depend on the integrity of the hippocampus (Winocur \& Moscovitch, 2011). Cognitive map theory also proposes that spatial memory is stored permanently in the hippocampus (O'Keefe and Nadel, 1978). The present findings favour the latter two theories, as activity of the hippocampus did not decrease between one day and 30 days retention of the water maze. In contrast, activity of the hippocampus actually increased from one day retention to 7 and 14 day retention, as measured by c-Fos and Arc expression. The results are consistent with previous studies which have shown equivalent or increased levels of IEG expression in the hippocampus from recent to remote memory retention of the water maze (Lopez et al., 2012; Bonaccorsi et al., 2013), but this is the first study to demonstrate a consistent pattern across three IEGs. IEG expression in the hippocampus has been shown to decrease during remote retention of spatial discrimination memory (Maviel et al., 2004), and is unclear what aspects of the water maze task render it so dependent upon the hippocampus for successful performance, whether hippocampal lesions result in a loss of specific spatial information (Riedel et al., 1999), or a more general impairment in navigation. Experiments conducted with rats outside the water maze in a complex "village" reveal that spatial memory in this environment can survive hippocampal lesions (Winocur, Moscovitch, Fogel, Rosenbaum, \& Sekeres, 2005), but when tasked with finding a novel route when usual paths are blocked, lesioned rats are significantly impaired (Winocur, Moscovitch, Rosenbaum, \& Sekeres, 2010). This suggests the hippocampus is necessary for the flexible use of a detailed spatial representation, which would explain the consistent impairments observed following hippocampal inactivation in the water maze regardless of the age of the memory, in contrast with less demanding spatial tasks, and the persistent neural activity observed in the hippocampus across the four retention time-points in the present study. The results are also consistent with those observed in a contextual fear paradigm, where the necessity of the dorsal hippocampus for the expression of remote memory has been demonstrated (Goshen et al., 2011). Furthermore, the hippocampus is necessary for reinstatement of the precise contextual memory (Cullen et al., 2015), accompanied by patterns of c-Fos activity which correlate with memory precision (Ruediger et al., 2011). Optogenetic inactivation of area CA1 during remote retention not only impairs contextual memory, but also results in the reduction of c-Fos activity in the anterior cingulate cortex (Goshen et al., 2011), suggesting the coactivation of these areas is required for the persistence of long-term memory, which supports the consistent hippocampal activation observed across all time-points in this study.

Of note, levels of Arc protein were undetectable in area CA1 of the hippocampus at any retention time point. While this is somewhat surprising given that a similar study (Gusev, Cui, Alkon, \& Gubin, 2005) observed Arc expression following spatial memory retention. These authors found that Arc was strongly expressed at $24 \mathrm{~h}$ post-retention, although there was little or no expression when examined at one-month retention. One explanation for the difference between studies is that Gusev et al. (2005) used Arc
mRNA rather than protein, which may be a more sensitive marker. Another unexpected finding in this study was the relatively high levels of expression in the caged control group, particularly when compared with the one day group, with significantly higher levels in the dentate gyrus suggesting a suppression of IEG activity during recent retention. One possible explanation could be provided by Lonergan, Gafford, Jarome, and Helmstetter (2010), who observed in a fear conditioning paradigm, after an initial task-related increase of Zif268 in the hippocampus, a subsequent drop below caged control levels for up to $24 \mathrm{~h}$. Given that recent retention took place $24 \mathrm{~h}$ following the final day of training in the water maze, this muted expression of Zif268 in the hippocampus may be a protective mechanism against interference during the earliest stages of consolidation.

Brain structures supporting remote spatial memory representations other than the anterior cingulate cortex and hippocampus have received little attention. The present study demonstrated that the entorhinal, perirhinal, retrosplenial and parietal cortex were increasingly recruited in accordance with the age of the spatial memory, which corroborates the results of a limited number of lesion, inactivation and electrophysiological studies which have been carried out in these regions. Lesions of the entorhinal cortex impair retention of the water maze (Hardman et al., 1997), possibly due to disruption of general location information (Hebert \& Dash, 2004), and the discovery of spatially selective cells in the medial entorhinal cortex highlights this region in particular as a likely candidate in the storage of long-term spatial memory (Fyhn, Molden, Witter, Moser, \& Moser, 2004). Lesions of the direct entorhinal projection to area CA1 in the hippocampus for up to three weeks following learning of the water maze task impairs retention, further emphasising a role for the entorhinal cortex in systems consolidation processes (Remondes \& Schuman, 2004). Rats with retrosplenial cortex lesions are slightly impaired during water maze acquisition, however they show no preference for the correct quadrant during a retention probe test (van Groen, Kadish, \& Wyss, 2004). Rats with retrosplenial lesions are significantly impaired one month, but not one week after training in a spatial discrimination task (Haijima \& Ichitani, 2008), further emphasising its role in long-term spatial memory. Lesions of the perirhinal cortex do affect spatial memory retention, but only over longer time periods, and its role appears to be specific to memory retrieval (Ramos \& Vaquero, 2005) rather than consolidation processes (Ramos, 2008). The similarity between patterns of IEG activity emerging in these regions from recent to remote memory and that of the medial prefrontal cortex suggests the successful retention of remote spatial memory becomes increasingly dependent upon the co-activation of these cortical regions over time.

The design of the current study also allowed us to investigate the extent to which three well established markers of neural activity exhibited similar expression over the course of consolidation. While there was a close correspondence in patterns of activity in the medial prefrontal cortex, the observed increases in hippocampal activity as measured by Fos and Arc expression early during the consolidation period, as well as the delayed increases in other cortical regions over time, were not observed with Zif268. One possible explanation is the relatively high basal expression of Zif268 compared to other IEG's such as c-Fos, where constitutive levels in the absence of external stimulation is extremely low (Kaczmarek \& Chaudhuri, 1997). Consequently Zif268 may not be as sensitive a marker of neural activity in response to learning and memory tasks as c-Fos or Arc. Another question raised by these results is the interpretation of IEG expression following a memory retrieval task. Given the necessity of IEGs for synaptic plasticity and memory formation, is their expression triggered by memory retrieval simply a marker of neural activity? Given the role of Zif268 in reconsolidation (Lee, Everitt, \& Thomas, 2004), the equiv- 
alent activation observed across different retrieval time-points may reflect reconsolidation of the memory, whereas the striking increases in Fos and Arc may be more indicative of increased recruitment of relevant regions. The findings presented here suggest prudence should be exercised in the use and interpretation of IEGs as markers of neural activity in learning and memory tasks.

The results of this study suggest it may take between two weeks and one month for new spatial memories to stabilise in extrahippocampal structures. The hippocampus appears to be necessary for the retrieval of detailed spatial memory regardless of its age. The increased recruitment of parahippocampal and neocortical areas over time, suggest long-term spatial memory storage becomes gradually distributed over a wide range of brain structures.

\section{Acknowledgments}

This work was funded in part by the Irish Research Council, and an NUIM John and Pat Hume scholarship held by D.N.B.

\section{References}

Albasser, M. M., Poirier, G. L., \& Aggleton, J. P. (2010). Qualitatively different modes of perirhinal-hippocampal engagement when rats explore novel vs. familiar objects as revealed by c-Fos imaging. The European Journal of Neuroscience, 31, $134-147$.

Barry, D. N., \& Commins, S. (2011). Imaging spatial learning in the brain using immediate early genes: Insights, opportunities and limitations. Reviews in the Neurosciences, 22(2), 131-142.

Bonaccorsi, J., Cintoli, S., Mastrogiacomo, R., Baldanzi, S., Braschi, C., Pizzorusso, T., ... Berardi, N. (2013). System consolidation of spatial memories in mice: Effects of enriched environment. Neural Plasticity, 2013, 956312.

Broadbent, N. J., Squire, L. R., \& Clark, R. E. (2006). Reversible hippocampal lesions disrupt water maze performance during both recent and remote memory tests. Learning \& Memory, 13, 187-191.

Cullen, P. K., Gilman, T. L., Winiecki, P., Riccio, D. C., \& Jasnow, A. M. (2015). Activity of the anterior cingulate cortex and ventral hippocampus underlie increases in contextual fear generalization. Neurobiology of Learning and Memory, 124, $19-27$.

Cullinan, W. E., Herman, J. P., Battaglia, D. F., Akil, H., \& Watson, S. J. (1995). Pattern and time course of immediate early gene expression in rat brain following acute stress. Neuroscience, 64, 477-505.

Duncan, G. E., Johnson, K. B., \& Breese, G. R. (1993). Topographic patterns of brain activity in response to swim stress: Assessment by 2-deoxyglucose uptake and expression of Fos-like immunoreactivity. The Journal of Neuroscience: The Official Journal of the Society for Neuroscience, 13, 3932-3943.

Euston, D. R., Gruber, A. J., \& McNaughton, B. L. (2012). The role of medial prefrontal cortex in memory and decision making. Neuron, 76, 1057-1070.

Frankland, P. W., \& Bontempi, B. (2005). The organization of recent and remote memories. Nature Reviews Neuroscience, 6, 119-130.

Frankland, P. W., O’Brien, C., Ohno, M., Kirkwood, A., \& Silva, A. J. (2001). AlphaCaMKII-dependent plasticity in the cortex is required for permanent memory. Nature, 411, 309-313.

Fyhn, M., Molden, S., Witter, M. P., Moser, E. I., \& Moser, M. B. (2004). Spatial representation in the entorhinal cortex. Science, 305, 1258-1264.

Goshen, I., Brodsky, M., Prakash, R., Wallace, J., Gradinaru, V., Ramakrishnan, C., \& Deisseroth, K. (2011). Dynamics of retrieval strategies for remote memories. Cell, 147, 678-689.

Gusev, P. A., Cui, C., Alkon, D. L., \& Gubin, A. N. (2005). Topography of Arc/Arg3.1 mRNA expression in the dorsal and ventral hippocampus induced by recent and remote spatial memory recall: Dissociation of CA3 and CA1 activation. The Journal of Neuroscience: The Official Journal of the Society for Neuroscience, 25, 9384-9397.

Guzowski, J. F., McNaughton, B. L., Barnes, C. A., \& Worley, P. F. (1999). Environment-specific expression of the immediate-early gene Arc in hippocampal neuronal ensembles. Nature Neuroscience, 2, 1120-1124.

Haijima, A., \& Ichitani, Y. (2008). Anterograde and retrograde amnesia of place discrimination in retrosplenial cortex and hippocampal lesioned rats. Learning E' Memory, 15, 477-482.

Hardman, R., Evans, D. J., Fellows, L., Hayes, B., Rupniak, H. T., Barnes, J. C., \& Higgins, G. A. (1997). Evidence for recovery of spatial learning following entorhinal cortex lesions in mice. Brain Research, 758, 187-200.

Harvey, D. R., McGauran, A. M., Murphy, J., Burns, L., McMonagle, E., \& Commins, S. (2008). Emergence of an egocentric cue guiding and allocentric inferring strategy that mirrors hippocampal brain-derived neurotrophic factor (BDNF) expression in the Morris water maze. Neurobiology of Learning and Memory, 89 (4), 462-479.
Hebert, A. E., \& Dash, P. K. (2004). Nonredundant roles for hippocampal and entorhinal cortical plasticity in spatial memory storage. Pharmacology, Biochemistry and Behavior, 79, 143-153.

Kaczmarek, L., \& Chaudhuri, A. (1997). Sensory regulation of immediate-early gene expression in mammalian visual cortex: Implications for functional mapping and neural plasticity. Brain Research. Brain Research Reviews, 23, 237-256.

Knapska, E., \& Kaczmarek, L. (2004). A gene for neuronal plasticity in the mammalian brain: Zif268/Egr-1/NGFI-A/Krox-24/TIS8/ZENK? Progress in Neurobiology, 74, 183-211.

Lee, J. L., Everitt, B. J., \& Thomas, K. L. (2004). Independent cellular processes for hippocampal memory consolidation and reconsolidation. Science, 304, 839-843.

Leon, W. C., Bruno, M. A., Allard, S., Nader, K., \& Cuello, A. C. (2010). Engagement of the PFC in consolidation and recall of recent spatial memory. Learning \& Memory, 17, 297-305.

Lonergan, M. E., Gafford, G. M., Jarome, T. J., \& Helmstetter, F. J. (2010). Timedependent expression of Arc and zif268 after acquisition of fear conditioning. Neural Plasticity, 2010, 139891.

Lopez, J., Herbeaux, K., Cosquer, B., Engeln, M., Muller, C., Lazarus, C., ... de Vasconcelos, A. P. (2012). Context-dependent modulation of hippocampal and cortical recruitment during remote spatial memory retrieval. Hippocampus, 22, $827-841$.

Maviel, T., Durkin, T. P., Menzaghi, F., \& Bontempi, B. (2004). Sites of neocortical reorganization critical for remote spatial memory. Science, 305, 96-99.

Morris, R. G. (1981). Spatial localization does not require the presence of local cues. Learning and Motivation, 12, 239-260.

Moser, E., Moser, M. B., \& Andersen, P. (1993). Spatial learning impairment parallels the magnitude of dorsal hippocampal lesions, but is hardly present following ventral lesions. The Journal of neuroscience: The Official Journal of the Society for Neuroscience, 13, 3916-3925.

Nadel, L., \& Moscovitch, M. (1997). Memory consolidation, retrograde amnesia and the hippocampal complex. Current Opinion in Neurobiology, 7, 217-227.

O'Keefe, J., \& Nadel, L. (1978). The hippocampus as a cognitive map. Oxford, UK: Clarenden Press.

Ons, S., Marti, O., \& Armario, A. (2004). Stress-induced activation of the immediate early gene Arc (activity-regulated cytoskeleton-associated protein) is restricted to telencephalic areas in the rat brain: Relationship to c-fos mRNA. Journal of Neurochemistry, 89, 1111-1118.

Paxinos, G., \& Watson, C. (2007). The rat brain in stereotaxic coordinates. London, UK: Elsevier.

Plath, N. et al. (2006). Arc/Arg3.1 is essential for the consolidation of synaptic plasticity and memories. Neuron, 52, 437-444.

Ramos, J. M. (2008). Perirhinal cortex lesions produce retrograde amnesia for spatial information in rats: Consolidation or retrieval? Learning \& Memory, 15, 587-596.

Ramos, J. M., \& Vaquero, J. M. (2005). The perirhinal cortex of the rat is necessary for spatial memory retention long after but not soon after learning. Physiology $\mathcal{E}$ Behavior, 86, 118-127.

Remondes, M., \& Schuman, E. M. (2004). Role for a cortical input to hippocampal area CA1 in the consolidation of a long-term memory. Nature, 431, 699-703.

Restivo, L., Vetere, G., Bontempi, B., \& Ammassari-Teule, M. (2009). The formation of recent and remote memory is associated with time-dependent formation of dendritic spines in the hippocampus and anterior cingulate cortex. The Journal of Neuroscience: The Official Journal of the Society for Neuroscience, 29 8206-8214.

Richards, B. A., Xia, F., Santoro, A., Husse, J., Woodin, M. A., Josselyn, S. A., et al. (2014). Patterns across multiple memories are identified over time. Nature Neuroscience.

Riedel, G., Micheau, J., Lam, A. G., Roloff, E. L., Martin, S. J., Bridge, H., ... Morris, R. G. (1999). Reversible neural inactivation reveals hippocampal participation in several memory processes. Nature Neuroscience, 2, 898-905.

Rudy, J. W., Biedenkapp, J. C., \& O'Reilly, R. C. (2005). Prefrontal cortex and the organization of recent and remote memories: An alternative view. Learning \& Memory, 12, 445-446.

Ruediger, S., Vittori, C., Bednarek, E., Genoud, C., Strata, P., Sacchetti, B., et al. (2011) Learning-related feedforward inhibitory connectivity growth required for memory precision. Nature, 473, 514-518.

Shimizu, E. (2000). NMDA receptor-dependent synaptic reinforcement as a crucial process for memory consolidation. Science, 290, 1170-1174.

Shires, K. L., \& Aggleton, J. P. (2008). Mapping immediate-early gene activity in the rat after place learning in a water-maze: The importance of matched control conditions. The European Journal of Neuroscience, 28, 982-996.

Squire, L. R. (1992). Memory and the hippocampus: A synthesis from findings with rats, monkeys, and humans. Psychological Review, 99, 195-231.

Squire, L. R., \& Bayley, P. J. (2007). The neuroscience of remote memory. Current Opinion in Neurobiology, 17, 185-196.

Teixeira, C. M., Pomedli, S. R., Maei, H. R., Kee, N., \& Frankland, P. W. (2006) Involvement of the anterior cingulate cortex in the expression of remote spatial memory. The Journal of Neuroscience: The Official Journal of the Society for Neuroscience, 26, 7555-7564.

Tischmeyer, W., \& Grimm, R. (1999). Activation of immediate early genes and memory formation. Cellular and Molecular Life Sciences: CMLS, 55, 564-574.

van Groen, T., Kadish, I., \& Wyss, J. M. (2004). Retrosplenial cortex lesions of area Rgb (but not of area Rga) impair spatial learning and memory in the rat Behavioural Brain Research, 154, 483-491.

Vetere, G., Restivo, L., Cole, C. J., Ross, P. J., Ammassari-Teule, M., Josselyn, S. A., et al (2011). Spine growth in the anterior cingulate cortex is necessary for the 
consolidation of contextual fear memory. Proceedings of the National academy of Sciences of the United States of America, 108, 8456-8460.

Weible, A. P. (2013). Remembering to attend: The anterior cingulate cortex and remote memory. Behavioural Brain Research, 245, 63-75.

Winocur, G., \& Moscovitch, M. (2011). Memory transformation and systems consolidation. Journal of the International Neuropsychological Society: JINS, 17, $766-780$
Winocur, G., Moscovitch, M., Fogel, S., Rosenbaum, R. S., \& Sekeres, M. (2005). Preserved spatial memory after hippocampal lesions: Effects of extensive experience in a complex environment. Nature Neuroscience, 8, 273-275.

Winocur, G., Moscovitch, M., Rosenbaum, R. S., \& Sekeres, M. (2010). An investigation of the effects of hippocampal lesions in rats on pre- and postoperatively acquired spatial memory in a complex environment. Hippocampus, 20, 1350-1365. 